\title{
EDITORIAL
}

\section{Thoracoscopic Surgery in the Esophageal Field}

\author{
Harushi Osugi, MD. \\ Director, Japanese Association for Thoracic Surgery, Osaka City University Graduate School of Medicine
}

Of gastrointestinal cancers, esophageal cancer is one of the most challenging diseases to perform prognosis. While we have enjoyed a recent advancement of radiation therapy and chemotherapy, surgical excision claims the highest success rate in curing esophageal cancer, if within a curable stage. This never-ending mission of improving cure rates, as of the mid-1980s, Japanese physicians have actively engaged in lymph node total dissection of such areas as the neck, thorax and abdomen, depending on where lymph node metastases have occurred. Compared to Europe and the USA, Japan sees strong demand for accurate, high quality lymph node total dissection, with a significant number of facilities conducting such lymph node total dissection.

How can we establish and maintain accuracy, high quality, and low-risk in developing complications? The answers must address surgeons' skills and how well the medical environment is maintained. Further, I believe an esophageal surgeon needs to be an accredited speciality board member as radical esophagectomy has often shown correlations among hospital volume, mortality and morbidity.

While our society launched the accredited speciality board system in both cardiovascular and pulmonary surgeries, establishment of the system for an esophageal field remains static. All the more reason the Japan Esophageal Society is working towards a speciality board system in esophageal surgery. Esophageal surgery requires specific skills, experiences, and environment, thus requiring an accreditation board. More rigorous procedure is anticipated for training surgeons to become an esophageal surgeon once the speciality board system is in place. This means higher quality treatment can be expected for patients who suffer from this disease.

Esophageal cancer is usually treated with a highly invasive, radical operation. Thoracoscopic esophagectomy was developed for the purpose of minimizing this

From Department of Gastroenterological Surgery, Osaka City University Graduate School of Medicine, Osaka, Japan.

Address for reprints: Harushi Osugi, MD, Department of Gastroenterological Surgery, Osaka City University Graduate School of Medicine, 1-4-3 Asahi-machi, Abeno-ku, Osaka 545-8585, Japan. invasiveness, and Cushieri et al. first reported the treatment with this method in 1992. In mid-1990, Europe and the USA published quite a few papers reporting on thoracoscopic esophagectomy. The majority of papers stated that while thoracoscopic surgery is capable of dissecting esophagus, its benefits have yet to be proven. Additionally, these papers concluded this method may not be worth a surgeon's increased level of stress. As a result, the papers on thoracoscopic esophagectomy have dwindled since 2000 .

I cannot help but to ask, "Is there no significance in conducting thoracoscopic esophagectomy?" Most papers that disapproved of thoracoscopic esophagectomy were written based on 30 or fewer cases. In contrast, 3 papers that were based on 80 or more cases concluded thoracoscopic esophagectomy was less invasive than open surgery, and thereby highly beneficial. What conclusion can we draw from this? I believe abundant research is absolutely essential to perform thoracoscopic esophagectomy. The same holds true for conventional radical operation on esophageal cancer.

Among scopic surgeries, laparoscopic cholecyctectomy is the most widely conducted surgery. The reasons are its ease of learning and performing, as well as its benefit of doing away with laparotomy that involves major invasive surgery. There seems little significance when deciding for or against thoracotomy as a radical operation on esophageal cancer that involves extensive invasiveness. There is great significance, however, in minimizing invasive procedures, providing an operation is performed in the best possible conditions.

Now, let me address some issues in performing radical thoracoscopic esophagectomy. First, this kind of procedure greatly demands surgeon expertise. Esophagectomy is ranked as one of the most difficult procedures and a physician needs to be accredited by the speciality board; plus it is managed thoracoscopically. Additionally, medical misadventures related to scopic procedures must be avoided.

In 2005, the Japan Society for Endoscopic Surgery launched the assessment and accreditation of operation skills for gastrointestinal surgery, including esophageal surgery, using video footage. Thoracoscopic esophagectomy remains incomplete in its definition: the broad 
interpretation includes a hand-assisted, and a "lookinginto procedure" that is similar to mini-thoracotomy, The Japan Society for Endoscopic Surgery excludes hand-assisted and "looking-into procedures" from thoracoscopic esophagectomy.

More pulmonary surgeries are conducted thoracoscopically compared with esophageal surgeries. The Japanese Association for Chest Surgery also excludes "looking-into surgery" from thoracoscopic surgery. In January 2006, we began conducting seminars for thoracoscopic surgery in several regions. We also plan to include a requirement of thoracoscopic surgery experiences in accrediting a pulmonary surgery specialist starting in 2009.
What are our society's missions as a governing body for thoracoscopic surgery? First, we should clarify the definition for existing techniques such as hand-assisted procedures and mini-thoracotomy. Second, we need to identify the advantages of each technique, such as ease of performing. Lastly, we should take advantage of the value scopic surgery yields; it can magnify microanatomy. Thus, we should clarify what is required to conduct microanatomy of mediastinum, and integrate techniques from both pulmonary and esophageal surgeries. Consequently, thoracoscopic surgery will continue to progress and utilization will be more widespread. This will lead to greater hope for thoracic disease patients. 